\title{
Liver Enzyme Elevations in Plasmodium falciparum Volunteer Infection Studies: Findings and Recommendations
}

\author{
Mohamed Farouk Chughlay, ${ }^{1 \star}$ Samantha Akakpo, ${ }^{1}$ Anand Odedra, ${ }^{2}$ Katalin Csermak-Renner, ${ }^{3}$ Elhadj Djeriou, ${ }^{4}$ Cornelis Winnips, ${ }^{3}$ \\ Didier Leboulleux, ${ }^{4}$ Aditya H. Gaur, ${ }^{5}$ G. Dennis Shanks, ${ }^{2}$ James McCarthy, ${ }^{2}$ and Stephan Chalon ${ }^{1}$ \\ ${ }^{1}$ Medicines for Malaria Venture, Geneva, Switzerland; ${ }^{2}$ QIMR Berghofer Medical Research Institute, Brisbane, Queensland, Australia; ${ }^{3}$ Novartis \\ Pharma AG, Basel, Switzerland; ${ }^{4}$ Sanofi, Gentilly, France; ${ }^{5}$ St. Jude Children's Research Hospital, Memphis, Tennessee
}

\begin{abstract}
Malaria volunteer infection studies (VISs) accelerate new drug and vaccine development. In the induced blood-stage malaria (IBSM) model, volunteers are inoculated with erythrocytes infected with Plasmodium falciparum. Observations of elevated liver enzymes in the IBSM model with new chemical entities (NCEs) promoted an analysis of available data. Data were reviewed from eight IBSM studies of seven different NCEs, plus two studies with the registered antimalarial piperaquine conducted between June 2013 and January 2017 at QIMR Berghofer, Brisbane, Australia. Alanine aminotransferase (ALT) was elevated ( $>2.5$ times the upper limit of normal [ $x U L N]$ ) in 20/114 (17.5\%) participants. Of these, 8.9\% (10/114) had moderate increases $(>2.5-5 \times U L N)$, noted in seven studies of six different NCEs \pm piperaquine or piperaquine alone, and $8.9 \%(10 / 114)$ had severe elevations (> $5 \times U L N)$, occurring in six studies of six different NCEs \pm piperaquine. Aspartate aminotransferase (AST) was elevated (>2.5 $\times$ ULN) in 11.4\% (13/114) of participants, across six of the 10 studies. Bilirubin was $>2 \times$ ULN in one participant. Published data from other VIS models, using sporozoite inoculation by systemic administration or mosquito feeding, also showed moderate/severe liver enzyme elevations. In conclusion, liver enzyme elevations in IBSM studies are most likely multifactorial and could be caused by the model conditions, that is, malaria infection/parasite density and/or effective parasite clearance, or by participant-specific risk factors, acetaminophen administration, or direct hepatotoxicity of the test drug. We make recommendations that may mitigate the risk of liver enzyme elevations in future VISs and propose measures to assist their interpretation, should they occur.
\end{abstract}

\section{INTRODUCTION}

Plasmodium falciparum volunteer infection studies (VISs), previously referred to as controlled human malaria infection (CHMI) studies, are conducted in non-endemic countries in healthy, malaria-naïve participants. Volunteer infection studies allow pharmacokinetic/pharmacodynamic profiling of new chemical entities (NCEs) to support early go/no-go decisions and enable optimized dose selection for Phase II trials, accelerating drug development.

There are two models of induced $P$. falciparum malaria infection. In the $P$. falciparum sporozoite (PfSPZ) challenge model, sporozoites are inoculated via mosquito bite or by systemic administration (intradermal, intramuscular, or intravenous). This model is generally used to investigate vaccine candidates and chemoprevention therapies, where infection suppression is the primary goal. Consequently, participants are exposed to low-level parasitemia, with early treatment using an appropriate antimalarial. In the induced blood-stage malaria (IBSM) model, malaria-infected erythrocytes are introduced intravenously. Used mainly for investigating NCE antimalarial activity against blood-stage parasitemia, that is, acute malaria treatment, this model has been predominantly conducted at QIMR-Berghofer Medical Research Institute Brisbane, Australia (QIMRB). ${ }^{1}$ To characterize drug parasite killing kinetics, IBSM generates higher parasitemia levels versus PfSPZ challenge, yet typically 1000 -fold lower than observed in uncomplicated malaria patients.

This investigation was prompted by observations of elevated alanine aminotransferase (ALT) and aspartate aminotransferase (AST) levels in IBSM studies conducted using

*Address correspondence to Mohamed Farouk Chughlay, Medicines for Malaria Venture (MMV), P.O. Box 1826, 20 Route de Pré-Bois, 1215 Geneva 15, Switzerland. E-mail: chughlayf@mmv.org several structurally diverse NCEs. Elevated ALT/AST was also observed with piperaquine, a component of the licensed antimalarial dihydroartemisinin-piperaquine, which has no clinical hepatotoxicity. Similar findings across these studies suggested there might be common factors in the IBSM model increasing the risk of transaminase elevation.

Hepatotoxicity is a major reason for drugs to fail clinical development. $^{2}$ Drug-induced liver injury (DILI) provokes liver test abnormalities or liver dysfunction, although diagnosis requires reasonable exclusion of other causes. ${ }^{3}$ Intrinsic DILI is caused when drugs affect hepatocytes, by a variety of dosedependent mechanisms, and can be predicted in human and animal models. ${ }^{3}$ By contrast, idiosyncratic DILI is rare, unpredictable, and dependent on factors inherent to the individual. ${ }^{3}$ Severe DILI is a leading cause of acute liver failure. ${ }^{3}$ To date, no genetic, metabolic, or other characteristic reliably predicts severe DILI in an individual. ${ }^{3}$ Increases in ALT/AST indicate hepatocellular injury but do not predict progression to severe DILI. Only when liver damage evolves to impair hepatocellular bilirubin excretion and serum bilirubin increases does functional impairment manifest clinically. ${ }^{3}$

The potential for a drug to cause severe DILI is described by Hy's law, that is, ALT elevation in a participant, usually substantial ( $\geq 3$ times the upper limit of normal $[\times U L N]$ ), seen concurrently with bilirubin $>2 \times$ ULN $^{3}$ Hy's law identifies a drug likely to cause severe DILI (fatal or requiring liver transplant) at a rate roughly $1 / 10$ the rate of Hy's law cases. Hy's law is confirmed if the drug caused the liver injury (excluding other causes), if hepatocytes are injured (not biliary obstruction, i.e., alkaline phosphatase [ALP] not substantially elevated), and if the injury was sufficient to impair liver function (hyperbilirubinemia). Finding two Hy's law cases causally related to drug administration in a clinical program indicates a high potential for severe DILI. ${ }^{3}$ Finding one case requires greater monitoring and investigation. 
Understanding the possible risks/consequences for healthy participants enrolled in IBSM Phase I studies is critical, especially when testing NCEs associated with liver safety signals in preclinical species. Also, safety is paramount for new antimalarial drugs and drug combination strategies. Consequently, the underlying mechanism of abnormal ALT/AST findings in IBSM studies and their relevance to drug development programs must be fully considered. This article examines the potential contributions of IBSM study procedures and the tested drugs to ALT/AST elevations in healthy human participants. We also consider recommendations for reducing the risk of ALT/AST elevations in VIS models and aiding their interpretation should they occur.

\section{METHODS}

Participants and design. All participants were healthy adults $\geq 18$ years old. Data were reviewed from eight studies of seven different NCEs conducted between June 2013 and January 2017 at QIMRB, Brisbane, Australia (Table 1). None of the NCEs had findings in preclinical or clinical studies suggesting hepatotoxic potential at the doses used in the IBSM model. Two studies (QP15C01 and QP15C05) included piperaquine administration to clear asexual parasites, while allowing gametocyte development, for assessment of NCE antigametocyte activity. However, in QP15C05, the planned NCE (OZ439) was not administered because ALT/AST levels increased after inoculation and piperaquine treatment. To further understand the potential mechanistic effects of the model on ALT/AST elevations, two additional studies with piperaquine alone were examined: QP13C05 and QP14C21 (Table 1).

All studies were conducted in accordance with the Declaration of Helsinki and the International Committee of Harmonization Good Clinical Practice Guidelines and received ethical approval from the Queensland Institute of Medical Research Human Research Ethics Committee and the ethical review boards of the study sponsors. All participants provided written informed consent.

Procedures. The general IBSM model is shown in Figure 1, with variations in the initial parasite inoculum and drug treatment day (Table 1). ${ }^{4}$ Adaptions were made to evaluate antigametocyte and transmission-blocking activity (Figure 1). ${ }^{5}$ The parasite inoculum was prepared as previously described, ${ }^{6}$ with between $\sim 1,800$ and 2,800 viable intraerythrocytic parasites ( $P$. falciparum 3D7) administered to each participant on day $0 .{ }^{4}$ Following establishment of infection, test drugs were administered on day 7 or 8 . Parasitemia was determined using real-time quantitative polymerase chain reaction (PCR), as previously described. ${ }^{7}$ Artemether-lumefantrine (Riamet ${ }^{\circledR}$, Novartis) was administered for recrudescence and as rescue therapy at the study end (Figure 1). Frequent blood samples were collected for drug pharmacokinetics and safety biochemistry and hematology laboratory assessments. Participants were confined and monitored for at least 36 hours post-drug administration with ambulatory follow-up until at least day 36 .

Retrospective microRNA-122 (miR-122) testing-a safety biomarker for liver cell damage ${ }^{8}$ - was performed for all participants in Study QP15C01 (ClinicalTrials.gov NCT02543086). Briefly, RNA was extracted from plasma using the QiAzol extraction method (Qiagen, Valencia, CA), and quantitative PCR analysis was conducted in accordance with the manufacturers' protocols (Biomark HD System, Fluidigm, San Francisco, CA).
For cDNA synthesis of plasma, the input was $3 \mu \mathrm{L}$ of the RNA preparations in a 5- $\mu \mathrm{L}$ reaction using Megaplex RT Primers Pool A (Life Technologies Corporation, Pleasanton, CA), with addition of a reverse transcriptase primer for mmu-miR-293 for normalization of miR-122 values. This method was validated with a limit of detection threshold value (Ct) for miR-122 of $23 \mathrm{Ct}$.

Data collection. Individual participant demographic data and parasitemia and liver enzyme test results were assembled from study statistical outputs. Baseline values for liver enzyme tests and total bilirubin were recorded on days -2 to 0 , before parasite inoculation. Parasitemia levels were determined on day 7 or 8 following inoculation, before treatment with NCE or piperaquine. Peak liver function tests, peak bilirubin, and peak parasitemia were defined as the highest value occurring after day 0 . Liver enzyme and bilirubin results were converted to values relative to the upper limit of normal (ULN) (Supplemental Table 1).

Data analysis and statistics. The WHO toxicity grading system was used to classify abnormal ALT, AST, and total bilirubin values relative to the ULN: mild (grade 1) 1.26-2.5 $\times$ ULN; moderate (grade 2) 2.6-5.0 $\times$ ULN; severe (grade 3) $5.1-10 \times$ ULN; and very severe (grade 4) > $10 \times$ ULN $^{9}$ Values $>2.5 \times$ ULN were considered potentially clinically important. Data analysis used individual participant data and descriptive statistics (MS Excel 2013, Microsoft Corp., Redmond, WA). A retrospective analysis was performed on the effect of acetaminophen on peak ALT/AST levels relative to the ULN using analysis of variance with multiple comparisons test. All enrolled participants who had parasitemia and were treated with an NCE and/or piperaquine were included in the analysis.

\section{RESULTS}

Participants. Baseline data for studies included in the QIMRB data review are shown in Table 2. Most participants were males (78.4\%; 91/116) and Caucasian (70.0\%; 80/116). Two participants in Study QP15C20 did not receive drug treatment and were excluded. Thus, the analysis population comprised 114 participants.

Liver enzyme elevations in the IBSM model. Alanine aminotransferase was elevated (> $2.5 \times$ ULN) in $17.5 \%(20 /$ 114) of participants across in eight studies, including seven different NCEs \pm piperaquine and piperaquine alone; AST elevations occurred in $11.4 \%$ (13/114) of participants, across six studies (Tables 1 and 3). The ALT:AST ratio was > 1 in 17/20 participants with ALT elevations; participants R104 (QP13C14), R103 (QP14C12), and R204 (QP14C12) had ALT: AST ratios < 1 (Table 1). There were no cases where AST was elevated without ALT elevation, whereas seven participants had ALT elevations without increased AST (Table 1, Figure 2).

In general, ALT started to increase on days 8-10 after inoculation (2-4 days after drug treatment), peaked at days 11-16, declining to normal levels by the study end (Figure 2A). Except for three participants with diverse profiles (R101(A), 5108, and R201), who each received different NCEs, the pattern of ALT elevations showed similar profiles. This suggests that the cause of ALT elevations was not drug specific. Aspartate aminotransferase elevations occurred mainly between days 10 and 16, although were delayed moderately for one participant and more obviously for four participants (Figure 2B). 
A



Malaria rescue medication

(artemether-lumefantrine)
End of study = Day 36
Recrudescence (sexual \& asexual)

B
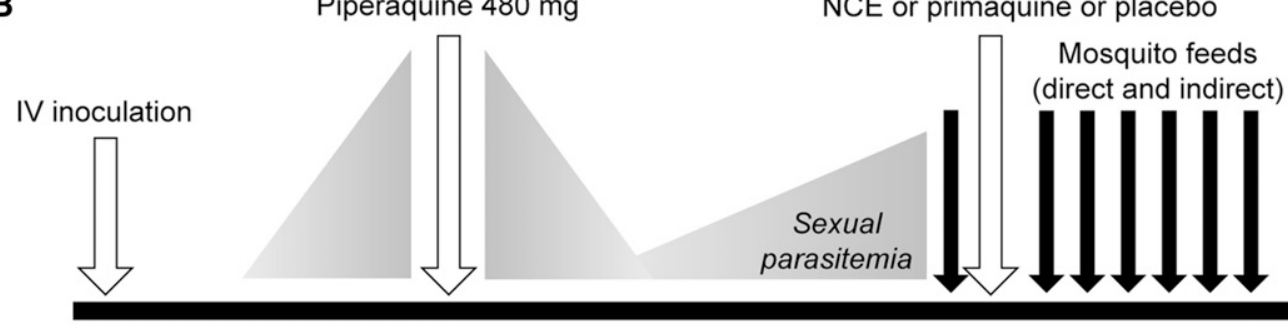

Day 0

Day 7 or 8

End of study = Day 36

C

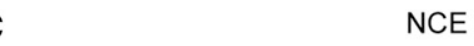

IV inoculation
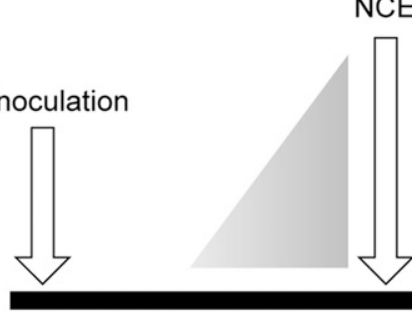

Piperaquine (to clear asexual stage)

Day 0

Day 7 or 8

End of study = Day 36

FIGURE 1. General method for induced blood-stage malaria studies. (A) Malaria infection is induced with an intravenous (IV) inoculum of parasitized erythrocytes. After 7-8 days, experimental treatment is given, and if recrudescence occurs, rescue therapy is given. (B) The system can be adapted to test for anti-gametocyte activity by administration of piperaquine before the new chemical entity (NCE) to enrich for gametocytes and remove blood-stage parasites; transmission blocking activity can also be tested via membrane feeding assays. (C) A combined experiment tests the NCE against blood-stage parasites at a subtherapeutic dose, then primaquine is given at recrudescence, followed by the NCE to test for antigametocyte activity.

In participant R103 (Study QP14C12 [OZ439/DSM265]), peak AST was 3 -fold higher than ALT, coinciding with elevations in lactate dehydrogenase $([\mathrm{LDH}] 4.8 \times \mathrm{ULN})$ and creatine kinase $(170.2 \times$ ULN). The participant commenced a weightlifting program 7 days before the biochemical abnormalities, after previously being inactive, and investigators attributed the findings to exercise.

Potential for severe DILI (Hy's law). To investigate the potential for severe liver injury as defined by Hy's law, ${ }^{3}$ an evaluation of drug-induced serious hepatotoxicity (E-DISH) 


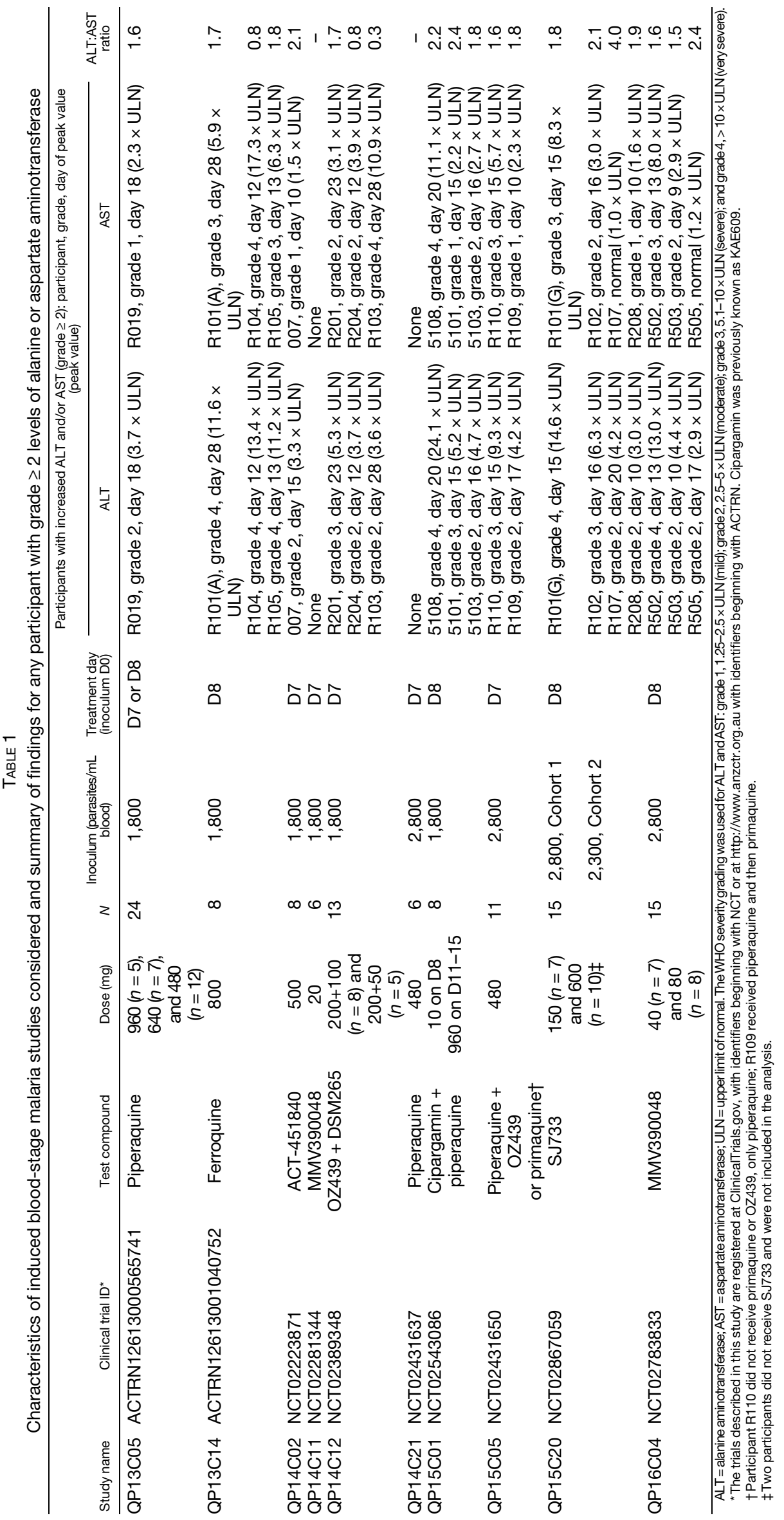






TABLE 3

Frequency of liver enzyme elevations in healthy participants treated with candidate antimalarial drugs or piperaquine in the induced blood-stage malaria model

\begin{tabular}{lcc}
\hline \multicolumn{1}{c}{ Severity } & ALT $(N=114)$ & AST $(N=114)$ \\
\hline Normal & $73(64.0)$ & $93(81.6)$ \\
Grade 1, mild (1.25-2.5 $\times$ ULN), $n(\%)$ & $21(18.4)$ & $8(7.0)$ \\
Grade 2, moderate $(2.6-5 \times$ ULN), $n(\%)$ & $10(8.8)$ & $5(4.4)$ \\
Grade 3, severe (5.1-10 $\times$ ULN), $n(\%)$ & $4(3.5)$ & $5(4.4)$ \\
Grade 4, very severe (> 10 $\times$ ULN), $n(\%)$ & $6(5.3)$ & $3(2.6)$ \\
\hline ALT = alanine aminotransferase; AST = aspartate aminotransferase; ULN = upper limit of \\
normal. Grades are based on the WHO Adverse Event Grading System
\end{tabular}

plot of ALT versus bilirubin relative to the ULN and a modified E-DISH plot (M-DISH) of changes in ALT and bilirubin relative to baseline was performed (Figure 3). Across the IBSM studies, there was one potential Hy's law case (participant 5103; Study QP15C01; cipargamin-piperaquine) (Figure 3). This 52year-old man had an undisclosed history of alcohol abuse, and a fatty liver and gallstones observed on ultrasound examination, performed after the ALT/AST elevations were identified. Baseline values (day 8) were mildly elevated for total and conjugated bilirubin $(1.4 \times \mathrm{ULN}$ and $1.3 \times \mathrm{ULN})$, ALT $(1.1 \times$ ULN), and gamma glutamyl transferase $(y G T)$ (1.5 $\times$ ULN) (Supplemental Figure 1). Peak elevations in ALT/ AST were not observed until 9 days after cipargamin administration and 1 day after piperaquine: AST 2.6 $\times$ ULN, ALT $4.7 \times$ ULN, and bilirubin $3.7 \times$ ULN. The participant received two $1-\mathrm{g}$ acetaminophen doses before peak ALT/AST. Total bilirubin mostly comprised unconjugated bilirubin. This was not suggestive of global liver function loss and inconsistent with DILI, being more likely explained by hemolysis or impaired bilirubin conjugation, as seen with Gilbert's syndrome. Subsequently, plasma miR-122 levels were assessed in all eight cohort participants; correlation between miR-122 and ALT profiles may reflect hepatocyte leakage or active release as a major cause for ALT elevations. ${ }^{8}$ In this case, the miR-122 profile did not match ALT, suggesting that bilirubin elevation was probably not caused by hepatocyte injury (Supplemental Figure 2). It was not possible to determine the mechanism underlying the liver function test abnormalities. Drug-induced liver injury expert review did not consider this a Hy's law case because of the bilirubin profile and history of alcohol abuse, and although a contribution for cipargamin-piperaquine could not be excluded, the cause was likely multifactorial.

Parasitemia. Across the IBSM studies, 19/20 cases of moderate-to-severe ALT elevation occurred when participants had peak parasitemia levels greater than 10,000 parasites $/ \mathrm{mL}$ (Figure 4). The relative risk of parasitemia $>10,000$ parasites $/ \mathrm{mL}$ for causing moderate/severe ALT increases was 4.8 (0.68-34.0, $P=0.116$ ) (Table 4). Parasite-induced hemolysis was also considered as a possible cause of ALT/AST elevations. Hemolysis index was increased in 29/114 participants, although the proportion of participants with an elevated hemolysis index was similar in those with moderate-to-severe ALT elevations $(30.0 \%[6 / 20])$ versus those with mild elevations/normal ALT (24.5\% [23/94]).

Further analysis was conducted on Study QP15C20 (SJ733), as different parasite inoculum levels were used in this study. Participants in Cohort $1(n=7)$ received a single $150 \mathrm{mg}$ SJ733 dose on day 8 , following inoculation with 2,800 P. falciparum on day 0 . Three participants from Cohort 1 (R101, R102, and R107) showed moderate-to-severe ALT/AST 

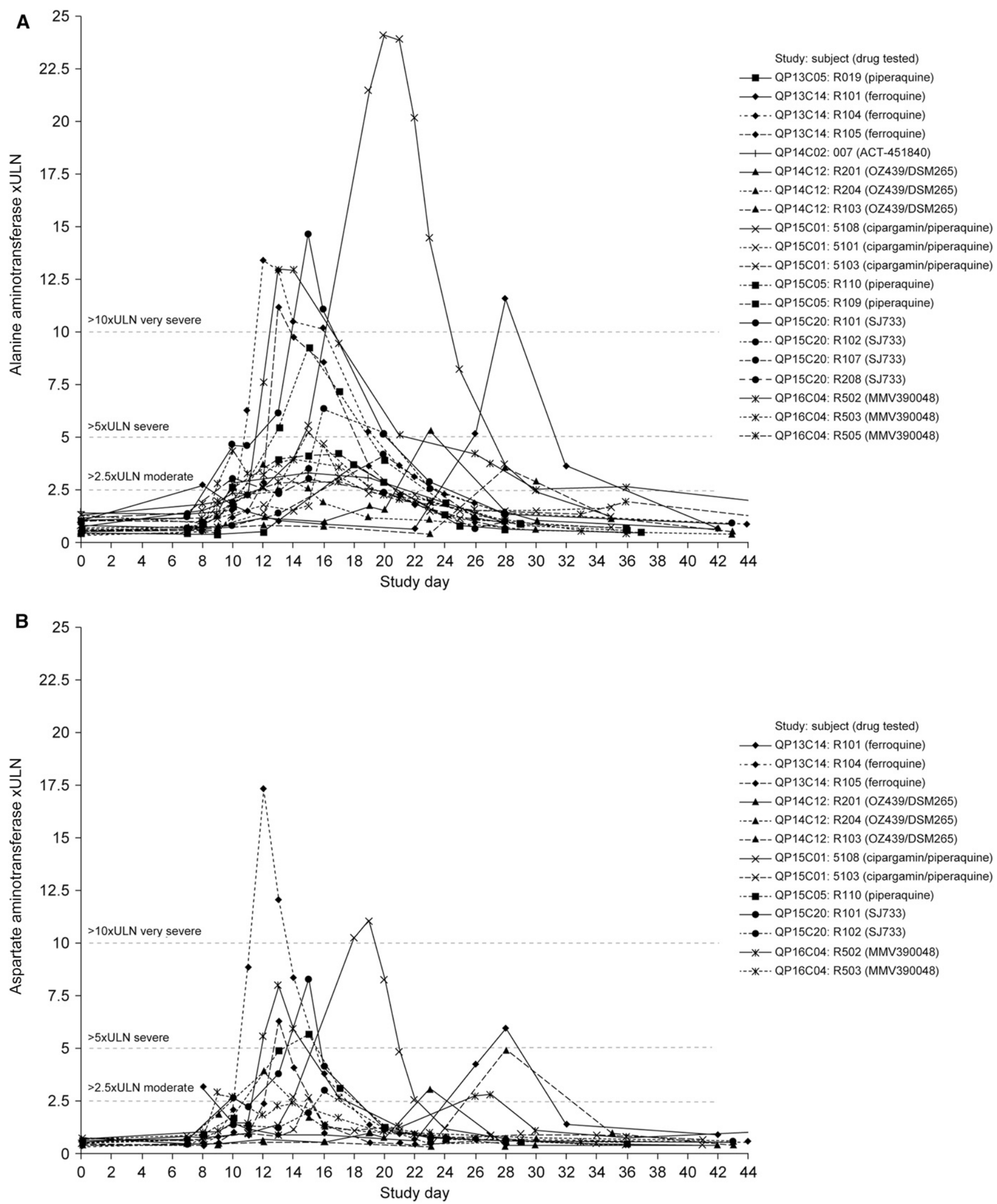

FIGURE 2. Overview of the time course of changes in liver transaminases for individual participants with moderate or severe increases $(\geq 2.6 \times \mathrm{ULN}$; WHO classification) in induced blood-stage malaria studies. (A) Alanine aminotransferase $(N=20)$. (B) Aspartate aminotransferase $(N=13)$. Malaria inoculum was given on day 0 and study drug administered on day 7 or 8 . 

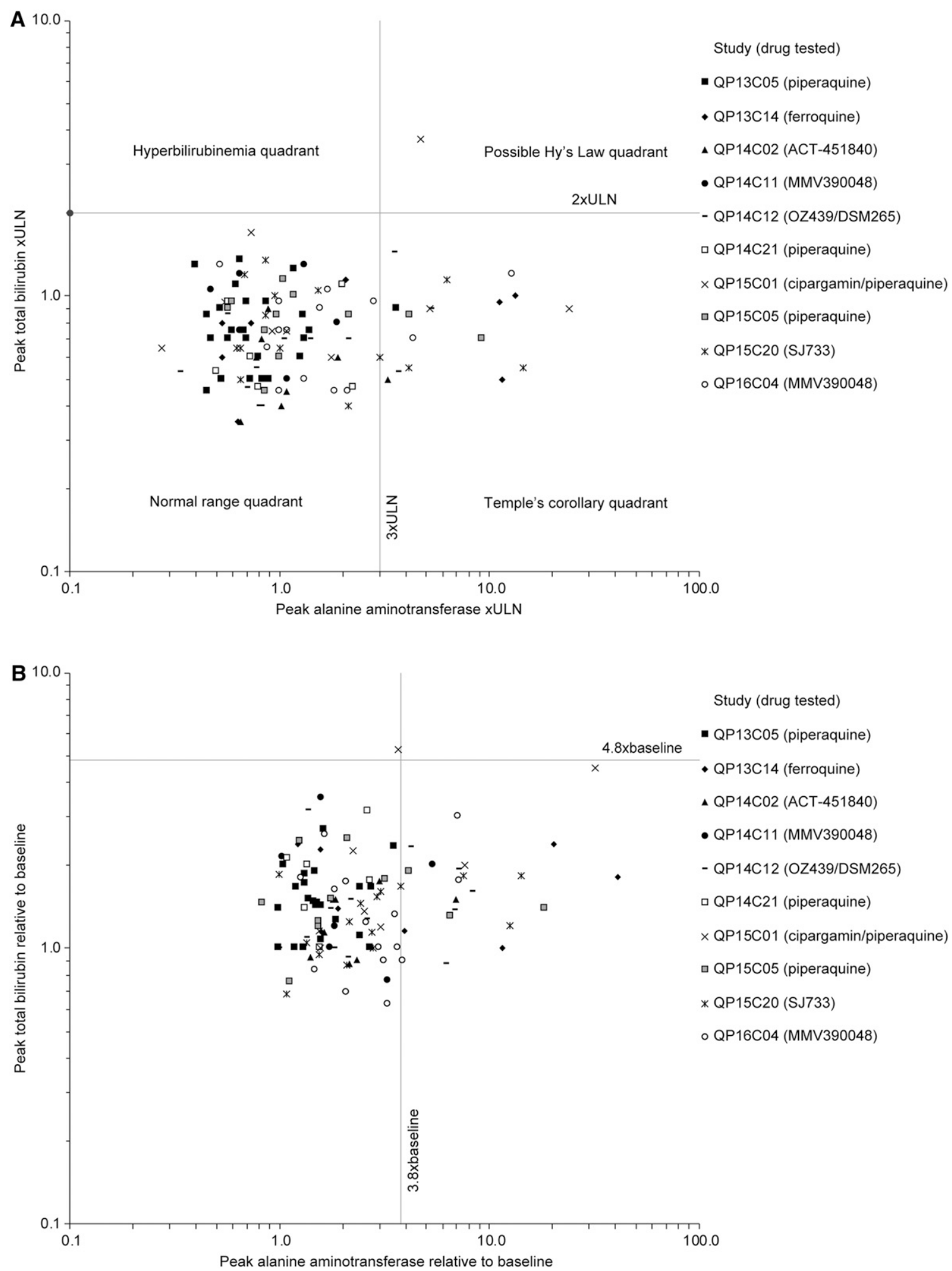

FIGURE 3. Peak alanine aminotransferase values relative to peak bilirubin levels for induced blood-stage malaria studies. (A) Absolute values normalized to the upper limit of normal (ULN). (B) Values relative to the baseline.

elevations, but no change in bilirubin, with relatively highpeak parasitemia levels $(147,074-378,077$ parasites $/ \mathrm{mL})$. As participants in Cohort $2(n=8)$ would receive a higher SJ733 dose (600 mg on day 8 ), they were inoculated with only 2,300 parasites. In Cohort 2, peak parasitemia levels were between
5,862 and 69,947 parasites/mL, with one participant (R208) having a moderate ALT elevation $(3.0 \times U L N)$. The greater antimalarial effects of the higher SJ733 dose in Cohort 2 resulted in more rapid parasite clearance versus Cohort 1 . In summary, compared with Cohort 1 where three participants 


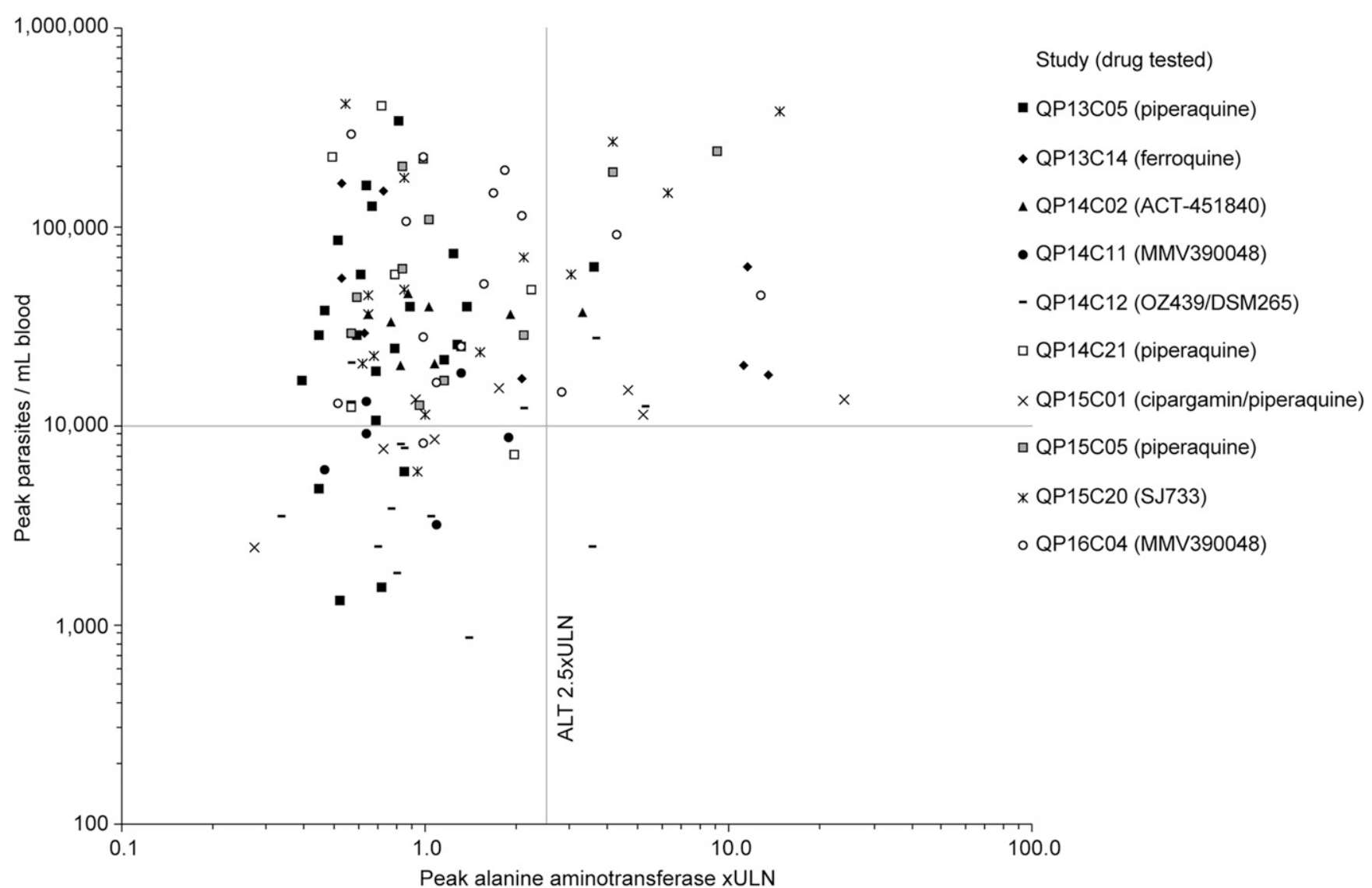

FIGURE 4. Relationship between peak parasitemia and alanine aminotransferase values in induced blood-stage malaria studies.

had AST/ALT elevations, in Cohort 2, a 4-fold higher dose with a greater antimalarial effect and a lower parasite inoculum size and peak parasitemia did not induce severe ALT/AST elevations.

A similar analysis was conducted for studies with the NCE MMV390048 (QP14C11 and QP16C04). In Study QP14C11 $(n=6)$, a 1,800 parasite inoculum with treatment on day 7 and a $20 \mathrm{mg}$ dose, caused a mild ALT increase in one participant (1.1 × ULN). In Study QP16C04, MMV390048 40 mg (Cohort B1) or $80 \mathrm{mg}$ (Cohort B2) was administered on day 8, with a 2,800 parasite inoculum. In Cohort B1 $(n=7)$, there were no ALT/AST elevations of grade 2 or higher. In Cohort B2 $(n=8)$, three participants showed ALT elevations; one severe (R502: $13.0 \times$ ULN; WHO grade 4) and two moderate (grade 2, R503: $4.4 \times$ ULN; R505: $2.9 \times$ ULN). This might appear to be a simple dose-response, with ALT/AST elevations more frequent at the higher MMV390048 dose. However, the same doses (40 and $80 \mathrm{mg}$ ) were tested in a Phase I study in healthy participants (ClinicalTrials.gov identifier: NCT02230579). In this case, there was no increase in ALT, with values similar to those obtained with placebo (Figure 5). This suggests that ALT increases were not a simple dose-response effect but related to the combination of malaria and drug.

Acetaminophen administration. Most participants $(59.6 \% ; 68 / 114)$ in the IBSM studies received acetaminophen for malaria symptoms. Administration started with symptom onset around day 6 , before test drug administration, and continued as required. In most cases, acetaminophen was given before ALT/AST increases were observed. In the six studies reporting severe ALT increases $(\geq 5.1 \times U L N), 6 / 10$ participants received acetaminophen at cumulative doses of 2 to $8.5 \mathrm{~g}$ (Supplemental Table 2). There was a statistically significant increase in peak ALT values relative to the ULN with cumulative acetaminophen doses $>6 \mathrm{~g}$ (mean $7.0 \times$ ULN [95\% $1.1,12.9])$ versus 0 to $\leq 6 \mathrm{~g}$ (mean $1.7 \times \operatorname{ULN}[95 \% \mathrm{Cl} 1.3,2.1])$ $(P<0.0005)$ (Figure 6). Adjustment for cohort and gender did not alter these results (data not shown). This translated into a significantly increased relative risk of ALT elevations with acetaminophen doses $>6 \mathrm{~g}$ versus lower doses or no acetaminophen (Table 4). Notably, relative risk further increased

TABLE 4

Relative risk of increased alanine aminotransferase with different cofounding factors in induced blood-stage malaria models

\begin{tabular}{|c|c|c|c|c|}
\hline Risk factors & Grade 2,3 , or $4 \operatorname{ALT}(n=20)$ & Normal or grade 1 ALT $(n=94)$ & Relative risk (95\% Cl) & $P$-value \\
\hline Parasitemia $\geq 10,000$ parasites $/ \mathrm{mL}$ & 19 & 72 & $4.8(0.68$ to 34.0$)$ & 0.116 \\
\hline Acetaminophen $>6 \mathrm{~g}$ & 4 & 6 & 2.6 (1.08 to 6.29$)$ & 0.034 \\
\hline $\begin{array}{l}\text { Parasitemia } \geq 10,000 \text { parasites } / \mathrm{mL} \text { plus } \\
\text { acetaminophen }>6 \mathrm{~g}\end{array}$ & 4 & 5 & 2.9 (1.24 to 6.88$)$ & 0.015 \\
\hline
\end{tabular}

$\mathrm{ALT}=$ alanine aminotransferase. 


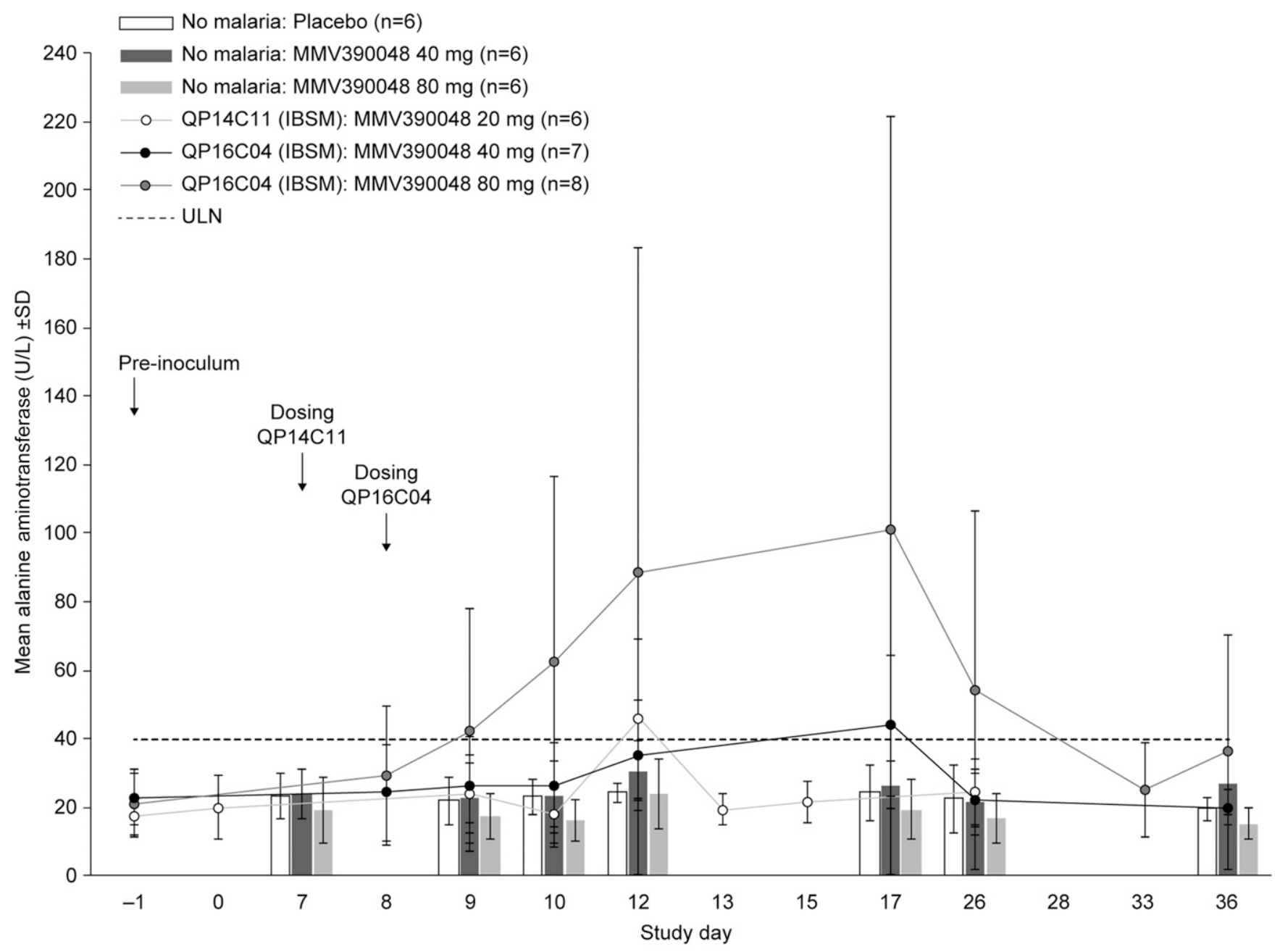

FIGURE 5. Mean alanine aminotransferase values following a single oral dose of MMV390048 (40 mg or $80 \mathrm{mg}$ ) given to participants with previous malaria inoculation (lines) or without malaria inoculation (bars). Inoculum intravenous administration was performed on Day 0.

among participants with acetaminophen doses $>6 \mathrm{~g}$ and parasitemia $>10,000$ parasites $/ \mathrm{mL}$ blood (Table 4).

Following the initial cases of ALT/AST elevations in studies QP13C14 and QP15C01, further investigation of samples from Study QP15C01 (cipargamin) showed an apparent temporal correlation between acetaminophen dosing and acetaminophen adduct levels (Supplemental Figure 3). However, the observed acetaminophen adduct levels $(\leq 0.1 \mu \mathrm{mol} / \mathrm{L})$ were not in the range associated with clinically relevant liver toxicity.

Piperaquine. Piperaquine data were examined because it has no known DILI risk, despite extensive use in acute uncomplicated malaria. ${ }^{10}$ In Study QP15C01, NCE (cipargamin) was given on day 8 at subtherapeutic doses for pharmacokinetic/ pharmacodynamic evaluation. Piperaquine $480 \mathrm{mg}$ was administered 3-7 days after cipargamin to clear asexual parasites, while permitting gametocyte emergence. Following intentional evaluation of low-dose $(10 \mathrm{mg})$ primaquine in the initial cohort to observe recrudescence, a repeat therapeutic dose of cipargamin was planned to test anti-gametocyte activity in the same participants. However, this repeat cipargamin dose was not given, as increases in ALT/AST were observed in 3/8 participants in Cohort 1 (5101, 5103, and
5108), requiring early study termination. The predominant effect was on ALT, but AST was also elevated, with levels rising after PCR-documented recrudescence and shortly after piperaquine administration. Participant 5103 had additional risk factors for ALT/AST increases, as discussed earlier, but in all three participants, the elevation in liver transaminases accelerated following piperaquine administration. Participants 5101 and 5108 had matching miR-122 and ALT profiles (Supplemental Figure 2), and hepatocyte leakage/release was assumed as the underlying reason for ALT elevations; reduced ALT clearance could not be the only cause. The other five participants in the cohort, who had no liver signals, had normal miR-122 levels. The ALT/AST increases following piperaquine appeared to be associated with parasitemia reduction by an effective antimalarial drug.

Three additional piperaquine studies were examined. In QP15C05, 2/12 participants showed ALT/AST elevations (R109: ALT $4.2 \times$ ULN, R110: $9.2 \times$ ULN) after 480 mg piperaquine administration on day 8 and a 2,800-parasite inoculum. QP13C05 had a lower inoculum size (1,800 parasites), with piperaquine 480-960 mg administered on day 7 or 8 , with one instance of moderately increased ALT (R019: ALT $3.7 \times$ ULN). In QP14C21, a piperaquine dose of $480 \mathrm{mg}$ on day 7 and an 


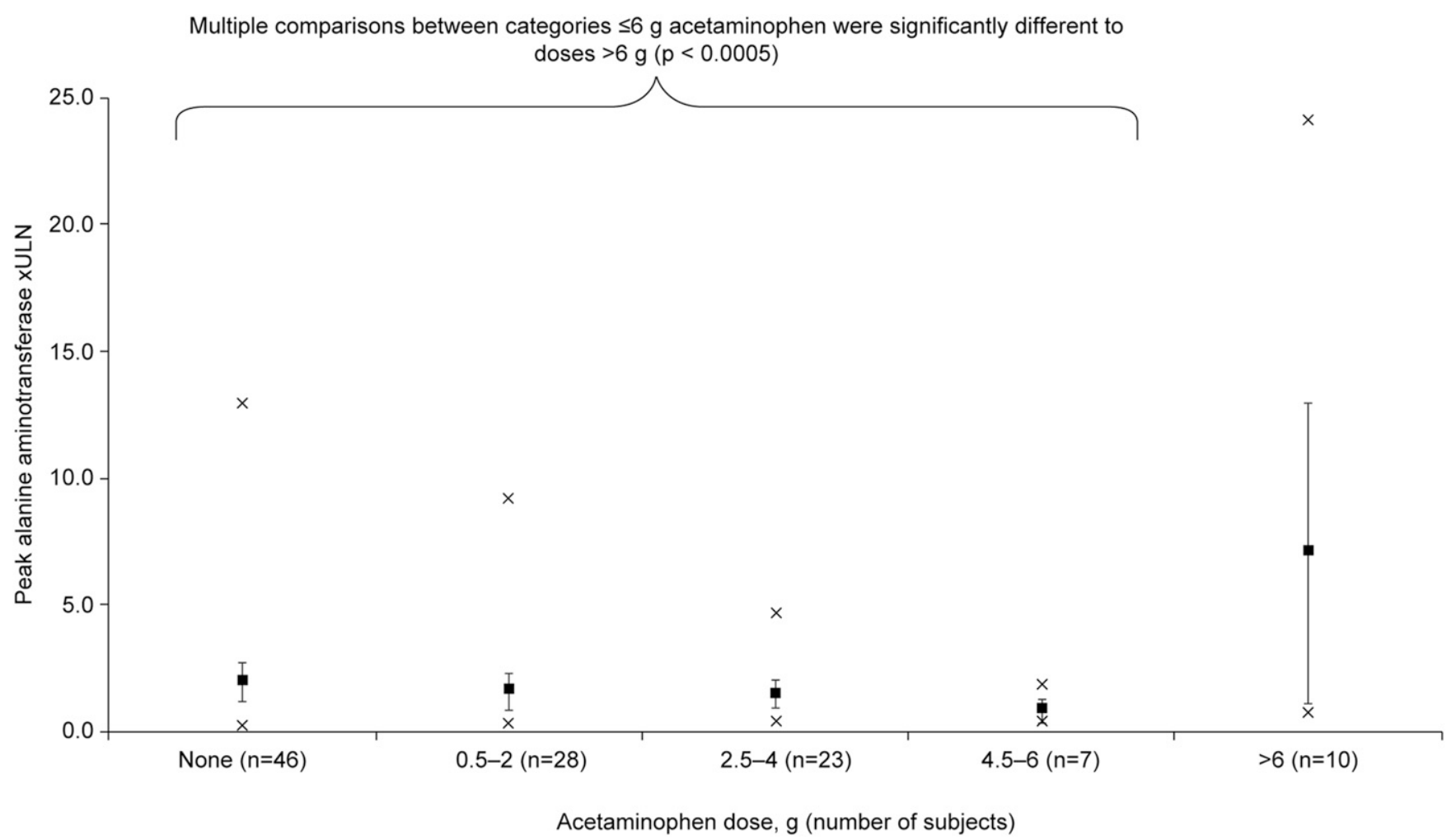

FIGURE 6. Relationship between cumulative acetaminophen dose and peak alanine aminotransferase levels relative to the upper limit of normal. Mean values $\pm 95 \% \mathrm{Cl}$; crosses are the maximum and minimum values.

inoculum of 2,800 parasites produced no ALT/AST elevations. Thus, earlier treatment (day 7$)$ and lower inoculum size $(1,800$ parasites) appeared to be protective against piperaquine induced ALT/AST elevations.

\section{ANALYSIS OF POTENTIAL CAUSES OF LIVER ENZYME ELEVATIONS IN THE IBSM MODEL}

Participant susceptibility. Across the studies examined, only one participant (5103; Study QP15C01 [cipargaminpiperaquine]) had elevated ALT and bilirubin (Figure 3), considered by DILI Experts to be caused by undisclosed alcohol abuse. One participant (R103; Study QP14C12 [OZ439/ DSM265]) had an AST increase co-incident with exercise, with typical increases in LDH and creatine kinase. Strenuous exercise is a known cause of liver enzyme increases, commonly observed in Phase I studies. ${ }^{11}$

The typical IBSM study protocol already excludes participants with known increased hepatotoxicity risk. However, additional participant-specific factors could predispose to liver injury during antimalarial drug treatment. These factors could be genetic, ${ }^{12}$ such as DILI caused by antituberculosis drugs in patients with the slow acetylator phenotype resulting from NAT2 variants, ${ }^{13,14}$ nonalcoholic steatohepatitis (NASH), ${ }^{15,16}$ or other conditions that would lead to exclusion but remain unknown or undisclosed by the participant. Age and gender may also play a role in DILI risk. ${ }^{17,18}$ However, apart from the two cases discussed, such participantspecific factors are an unlikely explanation for the high proportion of participants exhibiting increased ALT/AST in the IBSM studies described.
Presence of malaria. In Study QP15C20 (SJ733), a lower drug dose and higher inoculum size induced severe ALT/AST elevations, whereas a higher dose with a lower inoculum size did not. Similarly, in studies QP14C11 and QP16C04 (MMV390048), and in Study QP15C01 (cipargamin-piperaquine), the same doses that had no effect on ALT/AST levels in healthy participants without malaria caused ALT/AST elevations in healthy participants who were inoculated with malaria. Although there was a trend for ALT/AST elevations in participants with parasitemia levels > 10,000 parasites $/ \mathrm{mL}$, most participants (76.6\%; 72/94) without ALT elevations also had parasitemia levels above this level. Thus, although there may be some relationship between elevated liver enzymes and malaria in the IBSM data, it is not simply related to parasitemia level. Mild hemolysis occurred in some participants and dysregulation of iron detoxification is a possible cause of increased ALT/AST. ${ }^{19}$ Although there was no relationship between ALT/AST increases and hemolysis index, we cannot determine whether some participants were particularly sensitive to the effects of hemolysis, and so a relationship between hemolysis and ALT/AST increases cannot be excluded.

Adults and children living in endemic areas with acute uncomplicated $P$. falciparum malaria commonly experience mild ALT/AST elevations (up to $2.5 \times$ ULN; WHO Grading), and severe falciparum malaria is a known risk factor for liver injury. ${ }^{20,21}$ In the IBSM model, parasitemia in nonimmune participants is much lower at the time of test drug administration (mostly $<100,000$, rarely up to 400,000 parasites $/ \mathrm{mL}$ ) than observed for uncomplicated malaria in semi-immune patients from endemic areas (typically 1,000,000-100,000,000 parasites $/ \mathrm{mL}$ ). However, drug-induced parasite clearance 
kinetics in the IBSM model closely mimic those reported in malaria patients. ${ }^{1}$ Notably, direct inoculation of infected erythrocytes in the IBSM model eliminates the prepatent period for parasitemia development (i.e., liver stage of the disease). Most IBSM study participants experience some inflammation-related clinical symptoms, including malaise, chills, fever, headache, neck ache, myalgia, and back ache from day 7 onward. ${ }^{4}$ Thus, inflammation is evident after intravenous inoculation (day 0 ) at the point when drug treatment is initiated (day 7/8).

A possible explanation for transient ALT/AST elevations without impact on bilirubin in the IBSM model is that malaria inoculation induces acute inflammation and oxidative stress in healthy nonimmune participants. ${ }^{19}$ It is also possible that this inflammatory state could increase participant susceptibility to drug-induced effects on the liver from acetaminophen or test drugs or that there is some unappreciated individual predisposition to this phenomenon.

To better understand the impact of malaria on ALT/AST and bilirubin in nonimmune participants, a retrospective analysis was performed of hospitalized travelers with acute malaria returning from endemic areas to Queensland, Australia, between 2006 and $2016 .^{22}$ For this largely malaria-naïve population, ALT and/or AST elevations $\geq 3.1 \times$ ULN occurred in $15.1 \%(n=130 / 861)$ and $14.8 \%(127 / 861)$ of patients, respectively, with an incidence reported to day 7 following diagnosis. ${ }^{22}$ When stratifying by severity, ALT elevations were $\geq 3.1$ to $\leq 5.0 \times$ ULN in $8.8 \%(76 / 861)$ of patients, $\geq 5.1$ to $\leq 10 \times$ ULN in $4.7 \%(41 / 861)$, and $>10 \times$ ULN in $1.5 \%(13 / 861)$. The highest peak ALT elevation identified was $25.8 \times$ ULN on day 4 . Of the 130 cases with elevated ALT, 64.6\% (84/130) peaked between days 0 and 3 and $35.4 \%$ (46/130) between days 4 and 11; all largely resolved by day $28 .^{22}$ Note that these timings are synchronized with diagnosis, which occurred only after symptom onset, so cannot be directly compared with the current analysis where day 0 represents parasite inoculation.

Similarly, a recent review of 217 patients with imported uncomplicated $P$. falciparum malaria presenting at hospitals in the Netherlands between 2002 and 2017, showed moderate increases in liver function tests (ALT/AST/YGT/ALP) at admission in $14.0 \%(26 / 186)$, and severe increases in $4.8 \%$ $(9 / 186) .^{23}$ These increases were likely related to malaria hepatopathy. Notably, there was a positive association between parasite density on admission day and liver function test abnormalities $(r=0.25, P=0.005) .{ }^{23}$

Thus, it appears that up to $15 \%$ of malaria-naïve patients with imported uncomplicated $P$. falciparum malaria have ALT/AST increases, which may be related to parasitemia levels. These findings are consistent with the present analysis of participants in the IBSM model, that is, ALT increased in $17.5 \%$ (20/114) and AST increased in 11.4\% (13/114), with a trend for these to occur at higher parasite densities.

Acetaminophen administration. Acetaminophen has a known risk of dose-related intrinsic DILI, caused by acetaminophen-protein adduct formation. ${ }^{24}$ Acetaminophen causes pronounced ALT elevations in healthy participants with sub-chronic daily administration ( $4 \mathrm{~g} /$ day for $>4$ days), in a time frame consistent with those observed in the IBSM studies. ${ }^{25,26}$ In rodents, an induced inflammatory state lowers the toxicity threshold for several direct hepatotoxins, including acetaminophen. $^{27-29}$ It is, therefore, possible that malaria- associated inflammation could exacerbate this drug-induced effect. Alternatively, acetaminophen administration could be regarded as an indirect measure of inflammation and immune activation, higher doses likely being given to participants who had more malaria symptoms. None of the IBSM studies evaluated inflammatory markers, so this cannot be directly evaluated. It is also possible that acetaminophen increased sensitivity to NCE toxicity through additive depletion of glutathione by both drugs. Thus, acetaminophen could be considered as a risk factor for ALT elevation independently or in combination with malaria inoculation and/or test drugs.

Study drug. The ALT/AST elevations observed with piperaquine were unexpected as dihydroartemisinin-piperaquine is used to treat uncomplicated malaria without any evidence of a liver safety signal. This suggests that hepatotoxicity is not inevitably related to the study drugs. Also, it indicates that liver safety findings in IBSM studies do not necessarily translate into hepatotoxic risk in malaria patients. However, for the NCEs tested, their possible contribution to the observed liver toxicity cannot be excluded because of the absence of a control group.

Transient ALT/AST abnormalities are reported following antimalarial therapy of acute uncomplicated malaria and are severe in between 0.2 and $0.7 \%$ of patients. ${ }^{30}$ These changes are not associated with DILI and are not more common on repeated drug treatment. ${ }^{30,31}$ Severe ALT/AST increases following antimalarial therapy appear to occur more frequently in nonimmune versus semi-immune malaria patients ${ }^{22}$ and in children versus adults in malaria endemic regions. ${ }^{31}$ Whether there is a common underlying disease-related mechanism across antimalarial drug therapies which drives these ALT/ AST abnormalities and/or whether they result from direct drug-specific hepatotoxicity is unclear. These observations suggest that pharmacological treatment of acute uncomplicated malaria in itself is associated with additional stress for hepatocytes.

\section{DISCUSSION OF PRIOR EVIDENCE FOR LIVER ENZYME ELEVATIONS IN VOLUNTEER INFECTION STUDIES}

To further examine possible explanations for increased liver enzymes in the IBSM studies, we considered previous reports of ALT/AST elevations in VISs. This was not a formal systematic data review but aimed to extend the discussion of the observed liver enzyme elevations in the QIMRB IBSM studies. Relevant articles were identified by consultation with experts and investigators involved in VIS models. Also, a search was conducted of PubMed citations between June 1960 and September 2018 with the terms: challenge, inoculation, IBSM, CHMI, sporozoites, liver, toxicity, hepatotoxicity, malaria, aminotransferase, transaminases, challenge, and Plasmodium. Authors were not contacted for data sharing.

Induced blood-stage malaria studies. Most IBSM studies have been conducted at QIMRB. However, there are 12 published reports of IBSM studies conducted in the 1970 and 1980s by the United States of America Army. ${ }^{32-43}$ Unfortunately, only limited biochemistry data were available from two of these studies, and the incidence of ALT/AST elevations cannot be estimated. ${ }^{33,39}$

Sporozoite challenge model-Anopheles bite. Induction of malaria infection in human participants via the bites of infected Anopheles mosquitoes was first used to test antimalarial activity by the U.S. Army in the 1970s. It has since been 
used extensively for evaluating chemoprevention strategies with registered antimalarial drugs or NCEs.

A recent study examined the effect of marketed antimalarial drugs on liver function in healthy malaria-naïve participants aged 18-35 years participating in VISs using the PfSPZ challenge methodology, mainly the Anopheles bite model, conducted between 2001 and 2016 at Radboud University, the Netherlands. Liver function tests (ALT/AST/YGT/ALP) were moderately increased in $10.2 \%$ (19/187) of participants, with severe (grade 3 ) increases reported in $8.6 \%(16 / 187) .{ }^{23}$ Liver function test abnormalities peaked soon after treatment initiation, regardless of drug regimen, and returned to normal within 3 to 6 weeks. There was a positive association between liver enzyme elevations and parasite burden $(P<0.001)$, as well as cumulative inflammatory cytokine responses $(r=0.65, P=$ $0.008)$, and oxidative stress markers $(r=-0.63, P=0.001) .{ }^{23}$ These findings are, therefore, consistent with observations from the IBSM studies conducted at QIMRB, Australia.

In addition to the aforementioned review, we identified four reviews/meta-analyses with vaccine studies, ${ }^{44-47}$ a more recent vaccine study, ${ }^{48} 19$ drug studies, ${ }^{35-42,49-59}$ and six studies intended to validate/standardize methods for the PfSPZ Anopheles bite model. ${ }^{60-65}$ There was a surprising lack of data on ALT/AST levels; for the drug studies, 13/19 (68.4\%) publications omitted laboratory safety data.

One study with laboratory data included piperaquine. In a randomized trial of four antimalarial drug regimens to show feasibility of enriching for gametocytes, low-dose piperaquine (480 mg) or sulfadoxine-pyrimethamine $(500 \mathrm{mg} / 25$ $\mathrm{mg}$ ) were given, followed by a curative regimen on recrudescence. ${ }^{59}$ In the two low-dose piperaquine-containing regimens, ALT elevations (grade 2/3; WHO) occurred in $37.5 \%(3 / 8)$ of participants and AST elevations in the same proportion. Across all four regimens, ALT/AST elevations occurred in $33.3 \%(4 / 12)$ of participants. ${ }^{59}$ Thus, just as for the IBSM model, piperaquine administration during blood-stage malaria was associated with increases in liver enzymes in the PfSPZ Anopheles bite model.

Although placebo controls are often lacking in VISs, one randomized double-blind placebo-controlled chemoprevention study was conducted with pafuramidine. ${ }^{55}$ In this case, ALT/AST levels were elevated in 10/15 (66.6\%) healthy participants who developed malaria, of whom $3 / 5(60.0 \%)$ were in the placebo arm and $7 / 14(50.0 \%)$ in the pafuramidine arms. ${ }^{55}$ Four participants (1 placebo/3 pafuramidine) had ALT increases of WHO grade 3 severity $(5.1-10 \times U L N)$, with maximum increases of approximately $8 \times$ ULN (Supplemental Table 3$){ }^{55}$ The observation of moderate/severe ALT/AST elevations in placebo-controlled participants suggests a contribution of the study procedures (inoculation and/or acetaminophen).

Sporozoite challenge model-Systemic administration. Plasmodium falciparum sporozoite challenge by systemic administration uses infectious, aseptic, purified, cryopreserved $P$. falciparum NF54 sporozoites. The number administered varies according to the administration route: 3,200 intravenously and 75,000 by intramuscular injection, ${ }^{66}$ and although the number needed by intradermal administration has not been fully established, it exceeds $25,000 .^{67-69}$ Unlike the IBSM methodology, PfSPZ challenge includes a prepatent liver stage before parasitemia is detected and malaria symptoms emerge. Except for one study of chloroquine prophylaxis, ${ }^{70}$ and two recent studies evaluating chemoprevention with DSM265, ${ }^{58,71}$ antimalarial drug activity has not been tested in this model. Rather, approved antimalarial drugs are administered as rescue therapy immediately once malaria parasites are detected in blood smears or using PCR. Thus, parasitemia levels on the treatment day are usually low; generally $<10,000$ and often $<1,000$ parasites $/ \mathrm{mL}$.

We reviewed 14 publications of interest concerning PfSPZ challenge by systemic administration in nonimmune healthy participants. ${ }^{58,66-78}$ Laboratory safety data were often absent or insufficiently detailed during the timeframe of interest. When adverse events categorized under investigations or laboratory safety data were provided, aminotransferase elevations above the ULN were noted in nonimmune individuals at a rate of $0-61 \% .^{58,66,67,69,71-74,77}$ Severity grading information was not often available, although an ALT elevation of $16.6 \times$ ULN was noted in one study with atovaquoneproguanil. ${ }^{67}$ No data were available for acetaminophen dosing for any of the published studies.

\section{RECOMMENDATIONS}

Specific safety provisions for VISs with NCEs are proposed to minimize hepatotoxicity risk in study participants. Based on our analysis and published data, these provisions should be applied to both IBSM and PfSPZ models. Also, recommendations are made to maximize the opportunity to differentiate between potential DILI and model-related factors (Box 1). For drugs with hepatotoxic potential identified in preclinical species, a careful risk:benefit analysis is required. This might conclude that VISs be avoided or necessitate a modified design, for example, sequential enrolment to ascending doses following safety review.

Exclusion of high-risk participants. As the liver is involved in malaria, every precaution should be undertaken to exclude participants at greater risk of liver injury. Obtaining a minimum of two baseline samples before malaria inoculation at a 48- to 72-hour interval should identify participants with fluctuating ALT/AST exceeding $>1.25 \times$ ULN. Mild baseline ALT/AST elevations can indicate underlying hepatic stress, for example, caused by NASH or alcoholism. ${ }^{79}$ Induced blood-stage malaria study protocols should stipulate that ALT/AST elevations above acceptable values before the inoculum day should not be infected with malaria. After parasite inoculation, pretreatment ALT/AST and bilirubin levels should be obtained and reviewed by the investigator. Alanine aminotransferase/ AST may be tested up to 24 hours before planned NCE administration, and if above acceptable limits, standard of care (i.e., a

Box 1

Summary of recommendations

\begin{tabular}{|c|c|c|}
\hline$\#$ & Recommendation & Contributors \\
\hline 1 & Exclusion of high-risk participants & $\mathrm{Pl}$ and sponsor \\
\hline 2 & Enhanced informed consent process & $\mathrm{PI}$ and sponsor \\
\hline 3 & $\begin{array}{l}\text { Control group and comparable data from } \\
\text { Phase I studies }\end{array}$ & PI and sponsor \\
\hline 4 & $\begin{array}{l}\text { Control parasitemia levels at treatment } \\
\text { administration }\end{array}$ & PI, sponsor, and experts \\
\hline 5 & $\begin{array}{l}\text { Symptomatic treatment with ibuprofen } \\
\text { rather than acetaminophen }\end{array}$ & $\mathrm{PI}$ and sponsor \\
\hline 6 & Laboratory tests and biomarkers & PI, sponsor, and experts \\
\hline 7 & $\begin{array}{l}\text { Presentation of study results to standard } \\
\text { format }\end{array}$ & PI, and sponsor \\
\hline
\end{tabular}


registered antimalarial) can be administered instead of the NCE. If ALT/AST is elevated following inoculation and/or treatment, retesting should be conducted within 24 hours to exclude laboratory errors and reviewed for possible participant-dependent causes.

Enhanced informed consent process. Healthy participants enrolled in VISs are already informed that asymptomatic transient ALT/AST elevations may occur. Enhanced consent should highlight the potential severity of ALT/AST elevations and the consequences of withholding key information. Where an NCE is to be administered, alcohol, drugs of abuse, and more generally other medication, should be strictly avoided during the study and the follow-up period if possible. Alcohol and drugs of abuse screening tests with immediate reading (breath tests and urine dipstick, respectively) should be performed not just at screening, but at key time points, for example, pre-inoculation or pre-dose. Participants with a positive test should be withdrawn from the study, but will need treatment with a standard antimalarial if already inoculated with malaria.

Control group and comparable data from Phase I studies. A control arm, including either placebo or a registered antimalarial, would allow assessment of drug-dependent ALT/ AST elevations versus those caused by model conditions. Although a control group increases costs, potential delays in drug development while investigating elevated ALT/AST values are also costly. It may be possible to use an historical control group with a marketed antimalarial or placebo/rescue medication where all other covariates (inoculum size, treatment day, and concomitant medications) are equivalent. It would also be valuable to ensure that VIS design is comparable with the Phase I first-in-human study in terms of dose regimen and patient population. Thus, any liver signals from the VISs can be interpreted relative to similar participants who are not infected with malaria.

Control parasitemia levels at treatment administration. Although a threshold value has yet to be established, limiting parasitemia levels to $<10,000$ parasites $/ \mathrm{mL}$ blood would be precautionary. The feasibility of initiating NCE treatment at a predefined parasitemia threshold should be explored. Further work is needed to understand whether specific inflammatory/immune markers and/or malaria clinical score could be used to predict liver stress and integrated into a treatment decision tree. Parasite clearance kinetics for individual drugs, that is, fast or slow acting, could also influence the hepatic response to parasite clearance, and this requires further investigation.

Symptomatic treatment with ibuprofen rather than acetaminophen. Owing to the known effects of acetaminophen and adducts on hepatocytes, ibuprofen is suggested as an alternative for managing malaria symptoms after inoculation. Treatment with ibuprofen is more effective than acetaminophen in lowering temperatures in malaria patients throughout the first 4.5 hours after dosing. ${ }^{80}$ lbuprofen can be safely administered to healthy participants in a fasted state without increased risk of gastrointestinal toxicity. ${ }^{81}$ Alanine aminotransferase elevations can occur with ibuprofen doses of 2.4-3.2 g daily, but are generally mild. ${ }^{82}$ However, potential adverse effects, such as the known association of NSAIDs with acute kidney injury, ${ }^{83}$ requires specific evaluation in VISs.

Laboratory tests and biomarkers. A systematic liver safety panel, serum LDH, hemolysis panel, hemolysis index, and haptoglobin, along with parasite density and malaria clinical score should comprise VIS safety endpoints, with an appropriate decision tree if clinically relevant effects are detected. Observation of an ALT elevation > $5 \times$ ULN should prompt measurement of additional liver safety markers for mechanistic evaluation through a decision tree provided in the VIS protocol. For example, novel serum liver safety biomarkers investigated by Innovative Medicines Initiative-Safer and Faster Evidencebased Translation and Critical Path Institute's Predictive Safety Testing Consortium and endorsed by FDA/EMA could be considered. ${ }^{84-86}$ Biomarkers should be measured in the entire cohort, that is, including participants with and without elevated ALT/AST values, to allow comparison.

Presentation of study results to standard format. Summarized laboratory safety data with standardized severity gradings, along with parasitemia levels, malaria clinical score, and concomitant medications for all VIS participants should be included in preliminary datasets provided to the Safety Review Team, presented in clinical study reports, and published with manuscripts as Supplementary Data. Publications should state the adverse event severity grading system used. For any participants with elevated ALT/AST, all key parameters should be fully documented/reported, including the time course of changes in liver enzymes, parasitemia, medications received, malaria clinical score, and pharmacokinetic/pharmacodynamic data. A standardized set of tables and graphical outputs capturing the key findings and covariates is recommended. ${ }^{87}$ For comparability, data should be reported with reference to the ULN.

\section{CONCLUSION}

The evidence reviewed suggests that IBSM study procedures (i.e., presence of induced malaria and/or acetaminophen administration) may contribute to ALT/AST elevations in the IBSM model. Similar findings are reported from PfSPZ VIS models, both systemic administration and mosquito bite. However, based on the available evidence, the possibility that the test drugs could account for ALT/AST elevations cannot be formally excluded. The recommendations outlined include measures to minimize participant risk and aid the interpretation of findings suggestive of hepatotoxicity. Asymptomatic ALT/AST increases of limited severity should not have a major impact on NCE risk/benefit, or halt drug development. However, any findings suggestive of hepatotoxicity in VISs will need careful evaluation in Phase I trials and in comparison with existing antimalarial drugs in semi-immune malaria patients. In addition, the risk:benefit analysis of potentially hepatotoxic NCEs in VISs requires careful evaluation.

Received November 11, 2019. Accepted for publication March 9, 2020.

Published online April 20, 2020.

Note: Supplemental data, tables, and figures appear at www.ajtmh.org.

Acknowledgments: We acknowledge the contributions of Gerd A. Kullak-Ublick, John Pears, Michael Merz, and Karen I. Barnes for reviewing the manuscript and input during discussions with Terry Shapiro, Charles Wells, Isaie Reuling, Sophie Biguenet, and Stephan Duparc. We also thank Ria Woo and Emilie Rossignol for their dedicated support in collating the data used in this analysis and Peter O'Rourke for statistical advice. The trials described in this study are 
registered at ClinicalTrials.gov (identifiers: NCT02223871, NCT 02281344, NCT02389348, NCT02431637, NCT02543086, NCT02431650, NCT02867059, and NCT02783833) and at http://www.anzctr.org.au (identifiers: ACTRN12613000565741 and ACTRN12613001040752).

Financial support: This study was funded by Medicines for Malaria Venture.

Disclosure: Medical writing support was provided by Naomi Richardson, Magenta Communications Ltd., Abingdon, United Kingdom. M. F. C. and S. C. are employees of Medicines for Malaria Venture (MMV) and S. A. is a former employee; A. O., G. D. S., and J. M. are employees of QIMR Berghofer Medical Research Institute, which has received research funds from MMV, Novartis, and Sanofi; K. C.-R. and C. W. are employees of Novartis Pharma AG; E. D. is an employee of Sanofi and D. L. a former employee; A. H. G is an employee of St. Jude Children's Research Hospital, which has received research funds from Sanofi; A. H. G. reports grants from the Global Health Innovative Technology Fund (GHIT) during the conduct of the study; and St Jude Children's Research Hospital, where A. H. G. works, is the co-owner of the patent USP \#9,416,124 for the investigatory drug SJ733.

Authors' addresses: Mohamed Farouk Chughlay, Samantha Akakpo and Stephan Chalon, Research and Development, Medicines for Malaria Venture, Geneva, Switzerland, and UBC, Geneva, Switzerland, E-mails: chughlayf@mmv.org, samantha.akakpo@gmail.com, and chalons@mmv.org. Anand Odedra, Liverpool School of Tropical Medicine, School of Tropical Medicine, Pembroke Place, Liverpool, United Kingdom, and QIMR Berghofer Medical Research Institute, Clinical Tropical Medicine, Brisbane, Australia, E-mail: anand_tony@ hotmail.co.uk. Katalin Csermak-Renner and Cornelis Winnips, Research \& Development, Novartis AG, Basel, Switzerland, E-mails: katalin.csermak_renner@novartis.com and comelis.winnips@novartis.com. Elhadj Djeriou and Didier Leboulleux, Research \& Development, Sanofi, Paris, France, E-mails: elhadj.djeriou@sanofi.com and didierleboulleux@ gmail.com. Aditya H. Gaur, St. Jude Children's Research Hospital, Pharmacology, Memphis, TN, E-mail: aditya.gaur@stjude.org. G. Dennis Shanks, Australian Defence Force Malaria and Infectious Disease Institute, Enoggera, Australia, and University of Queensland School of Public Health, Brisbane, Australia, E-mail: dennis.shanks@defence.gov.au. James McCarthy, QIMR, Clinical Tropical Medicine, Herston, Australia, E-mail: j.mccarthy@uq.edu.au.

This is an open-access article distributed under the terms of the Creative Commons Attribution (CC-BY) License, which permits unrestricted use, distribution, and reproduction in any medium, provided the original author and source are credited.

\section{REFERENCES}

1. McCarthy JS et al., 2016. Linking murine and human Plasmodium falciparum challenge models in a translational path for antimalarial drug development. Antimicrob Agents Chemother 60: 3669-3675.

2. Weaver RJ, Valentin JP, 2019. Today's challenges to de-risk and predict drug safety in human "mind-the-gap". Toxicol Sci 167: 307-321.

3. U.S. Department of Health and Human Services, Food and Drug Administration, Center for Drug Evaluation and Research (CDER), Center for Biologics Evaluation and Research (CBER), 2009. Guidance for Industry: Drug-Induced Liver Injury: Premarketing Clinical valuation. Available at: www.fda.gov/ downloads/drugs/guidancecomplianceregulatoryinformation/ Guidances/UCM174090.pdf. Accessed September 23, 2018.

4. McCarthy JS et al., 2011. A pilot randomised trial of induced blood-stage Plasmodium falciparum infections in healthy volunteers for testing efficacy of new antimalarial drugs. PLoS One 6: e21914.

5. Collins KA et al., 2018. A controlled human malaria infection model enabling evaluation of transmission-blocking interventions. J Clin Invest 128: 1551-1562.

6. Cheng Q, Lawrence G, Reed C, Stowers A, Ranford-Cartwright L, Creasey A, Carter R, Saul A, 1997. Measurement of Plasmodium falciparum growth rates in vivo: a test of malaria vaccines. Am J Trop Med Hyg 57: 495-500.

7. Rockett RJ, Tozer SJ, Peatey C, Bialasiewicz S, Whiley DM, Nissen MD, Trenholme K, Mc Carthy JS, Sloots TP, 2011. A real-time, quantitative PCR method using hydrolysis probes for the monitoring of Plasmodium falciparum load in experimentally infected human volunteers. Malar J 10: 48.

8. Laterza OF, Scott MG, Garrett-Engele PW, Korenblat KM, Lockwood CM, 2013. Circulating miR-122 as a potential biomarker of liver disease. Biomark Med 7: 205-210.

9. World Health Organization, 1979. WHO Handbook for Reporting Results of Cancer Treatment. Available at: http://apps.who.int/ iris/bitstream/handle/10665/37200/WHO_OFFSET_48.pdf? sequence $=1$ \&isAllowed $=y$. Accessed January 16, 2019.

10. Zani B, Gathu M, Donegan S, Olliaro PL, Sinclair D, 2014. Dihydroartemisinin-piperaquine for treating uncomplicated Plasmodium falciparum malaria. Cochrane Database Syst Rev: CD010927.

11. Pettersson J, Hindorf U, Persson P, Bengtsson T, Malmqvist U, Werkstrom V, Ekelund M, 2008. Muscular exercise can cause highly pathological liver function tests in healthy men. $\mathrm{Br} \mathrm{J}$ Clin Pharmacol 65: 253-259.

12. Aithal GP, Grove Jl, 2015. Genome-wide association studies in drug-induced liver injury: step change in understanding the pathogenesis. Semin Liver Dis 35: 421-431.

13. Yuliwulandari $R$ et al., 2016. NAT2 variants are associated with drug-induced liver injury caused by anti-tuberculosis drugs in Indonesian patients with tuberculosis. J Hum Genet 61: 533-537.

14. Aithal GP, 2015. Pharmacogenetic testing in idiosyncratic druginduced liver injury: current role in clinical practice. Liver Int 35: 1801-1808.

15. Chalasani $\mathrm{N}$ et al., 2015. Features and outcomes of 899 patients with drug-induced liver injury: the DILIN prospective study. Gastroenterology 148: 1340-1352 e7.

16. Regev $A$ et al., 2019. Consensus: guidelines: best practices for detection, assessment and management of suspected acute drug-induced liver injury during clinical trials in patients with nonalcoholic steatohepatitis. Aliment Pharmacol Ther 49: 702-713.

17. Amacher DE, 2014. Female gender as a susceptibility factor for drug-induced liver injury. Hum Exp Toxicol 33: 928-939.

18. Liss G, Rattan S, Lewis JH, 2010. Predicting and preventing acute drug-induced liver injury: what's new in 2010? Expert Opin Drug Metab Toxicol 6: 1047-1061.

19. de Mast $Q$ et al., 2009. Mild increases in serum hepcidin and interleukin-6 concentrations impair iron incorporation in haemoglobin during an experimental human malaria infection. $\mathrm{Br}$ $J$ Haematol 145: 657-664.

20. Viriyavejakul P, Khachonsaksumet V, Punsawad C, 2014. Liver changes in severe Plasmodium falciparum malaria: histopathology, apoptosis and nuclear factor kappa B expression. Malar J 13: 106.

21. Anand AC, Puri $P, 2005$. Jaundice in malaria. J Gastroenterol Hepatol 20: 1322-1332.

22. Woodford J, Shanks G, Griffin P, Chalon S, McCarthy J, 2018. The dynamics of liver function test abnormalities following malaria infection: a retrospective observational study. Am J Trop Med Hyg 98: 1113-1119.

23. Reuling IJ et al., 2018. Liver injury in uncomplicated malaria is an overlooked phenomenon: an observational study. EBioMedicine 36: 131-139.

24. James LP, Letzig L, Simpson PM, Capparelli E, Roberts DW, Hinson JA, Davern TJ, Lee WM, 2009. Pharmacokinetics of acetaminophen-protein adducts in adults with acetaminophen overdose and acute liver failure. Drug Metab Dispos 37: 1779-1784.

25. Heard K, Green JL, Anderson V, Bucher-Bartelson B, Dart RC, 2014. A randomized, placebo-controlled trial to determine the course of aminotransferase elevation during prolonged acetaminophen administration. BMC Pharmacol Toxicol 15: 39.

26. Watkins PB, Kaplowitz N, Slattery JT, Colonese CR, Colucci SV, Stewart PW, Harris SC, 2006. Aminotransferase elevations in healthy adults receiving 4 grams of acetaminophen daily: a randomized controlled trial. JAMA 296: 87-93.

27. Maddox JF, Amuzie CJ, Li M, Newport SW, Sparkenbaugh E, Cuff CF, Pestka JJ, Cantor GH, Roth RA, Ganey PE, 2010. Bacterialand viral-induced inflammation increases sensitivity to 
acetaminophen hepatotoxicity. J Toxicol Environ Health A 73: 58-73.

28. Ganey PE, Luyendyk JP, Maddox JF, Roth RA, 2004. Adverse hepatic drug reactions: inflammatory episodes as consequence and contributor. Chem Biol Interact 150: 35-51.

29. Lu J, Jones AD, Harkema JR, Roth RA, Ganey PE, 2012. Amiodarone exposure during modest inflammation induces idiosyncrasy-like liver injury in rats: role of tumor necrosis factor-alpha. Toxicol Sci 125: 126-133.

30. Pryce J, Hine P, 2019. Pyronaridine-artesunate for treating uncomplicated Plasmodium falciparum malaria. Cochrane Database Syst Rev 1: CD006404.

31. West African Network for Clinical Trials of Antimalarial D, 2018. Pyronaridine-artesunate or dihydroartemisinin-piperaquine versus current first-line therapies for repeated treatment of uncomplicated malaria: a randomised, multicentre, openlabel, longitudinal, controlled, phase 3b/4 trial. Lancet 391: 1378-1390.

32. Cosgriff TM, Boudreau EF, Pamplin CL 3rd, Berman JD, Shmuklarsky MJ, Canfield CJ, 1984. Evaluation of the 4-pyridinemethanol WR 180,409 (enpiroline) in the treatment of induced Plasmodium falciparum infections in healthy, non-immune subjects. Am J Trop Med Hyg 33: 767-771.

33. Cosgriff TM, Boudreau EF, Pamplin CL, Doberstyn EB, Desjardins $\mathrm{RE}$, Canfield CJ, 1982. Evaluation of the antimalarial activity of the phenanthrenemethanol halofantrine (WR 171,669). Am J Trop Med Hyg 31: 1075-1079.

34. Rinehart J, Arnold J, Canfield CJ, 1976. Evaluation of two phenanthrenemethanols for antimalarial activity in man: WR 122,455 and WR 171,669. Am J Trop Med Hyg 25: 769-774.

35. Arnold JD, Martin DC, Carson PE, Rieckmann KH, Willerson D Jr., Clyde DF, Miller RM, 1973. A phenanthrene methanol (WR 33063) for treatment of acute malaria. Antimicrob Agents Chemother 3: 207-213.

36. McNamara JV, Rieckmann KH, Frischer H, Stockert TA, Carson PE, Powell RD, 1967. Acquired decrease in sensitivity to quinine observed during studies with a strain of chloroquine-resistant Plasmodium falciparum. Ann Trop Med Parasitol 61: 386-395.

37. Rieckmann KH, McNamara JV, Frischer H, Stockert TA, Carson PE, Powell RD, 1968. Gametocytocidal and sporontocidal effects of primaquine and of sulfadiazine with pyrimethamine in a chloroquine-resistant strain of Plasmodium falciparum. Bull World Health Organ 38: 625-632.

38. Rieckmann KH, Powell RD, McNamara JV, Willerson D Jr., Lass L, Frischer H, Carson PE, 1971. Effects of tetracycline against chloroquine-resistant and chloroquine-sensitive Plasmodium falciparum. Am J Trop Med Hyg 20: 811-815.

39. Williams RL, Trenholme GM, Carson PE, Frischer H, Rieckmann $\mathrm{KH}, 1978$. The influence of acetylator phenotype on the response to sulfalene in individuals with chloroquine-resistant falciparum malaria. Am J Trop Med Hyg 27: 226-231.

40. Williams RL, Trenholme GM, Carson PE, Frischer H, Rieckmann $\mathrm{KH}, 1975$. Acetylator phenotype and response of individuals infected with a chloroquine-resistant strain of Plasmodium falciparum to sulfalene and pyrimethamine. Am J Trop Med Hyg 24: 734-739.

41. Trenholme CM, Williams RL, Desjardins RE, Frischer $\mathrm{H}$, Carson PE, Rieckmann KH, Canfield CJ, 1975. Mefloquine (WR $142,490)$ in the treatment of human malaria. Science 190: 792-794.

42. Martin DC, Arnold JD, Clyde DF, al-lbrahim M, Carson PE, Rieckmann KH, Willerson D Jr., 1973. A quinoline methanol (WR 30090) for treatment of acute malaria. Antimicrob Agents Chemother 3: 214-219.

43. Powell RD, McNamara JV, 1970. Infection with chloroquineresistant Plasmodium falciparum in man: prepatent periods, incubation periods, and relationships between parasitemia and the onset of fever in nonimmune persons. Ann N Y Acad Sci 174: 1027-1041.

44. Church LW et al., 1997. Clinical manifestations of Plasmodium falciparum malaria experimentally induced by mosquito challenge. J Infect Dis 175: 915-920.

45. Verhage DF, Telgt DS, Bousema JT, Hermsen CC, van Gemert GJ, van der Meer JW, Sauerwein RW, 2005. Clinical outcome of experimental human malaria induced by Plasmodium falciparum-infected mosquitoes. Neth J Med 63: 52-58.

46. Epstein JE, Rao S, Williams F, Freilich D, Luke T, Sedegah M, de la Vega P, Sacci J, Richie TL, Hoffman SL, 2007. Safety and clinical outcome of experimental challenge of human volunteers with Plasmodium falciparum-infected mosquitoes: an update. J Infect Dis 196: 145-154.

47. Roestenberg M, de Vlas SJ, Nieman AE, Sauerwein RW, Hermsen CC, 2012. Efficacy of preerythrocytic and blood-stage malaria vaccines can be assessed in small sporozoite challenge trials in human volunteers. J Infect Dis 206: 319-323.

48. Rampling $T$ et al., 2018. Safety and efficacy of novel malaria vaccine regimens of RTS,S/AS01B alone, or with concomitant ChAd63-MVA-vectored vaccines expressing ME-TRAP. NPJ Vaccines 3: 49.

49. Brueckner RP, Coster T, Wesche DL, Shmuklarsky M, Schuster BG, 1998. Prophylaxis of Plasmodium falciparum infection in a human challenge model with WR 238605, a new 8-aminoquinoline antimalarial. Antimicrob Agents Chemother 42: 1293-1294.

50. Shapiro TA, Ranasinha CD, Kumar N, Barditch-Crovo P, 1999. Prophylactic activity of atovaquone against Plasmodium falciparum in humans. Am J Trop Med Hyg 60: 831-836.

51. Berman JD, Nielsen R, Chulay JD, Dowler M, Kain KC, Kester KE, Williams J, Whelen AC, Shmuklarsky MJ, 2001. Causal prophylactic efficacy of atovaquone-proguanil (Malarone) in a human challenge model. Trans R Soc Trop Med Hyg 95: 429-432.

52. Deye GA et al., 2012. Prolonged protection provided by a single dose of atovaquone-proguanil for the chemoprophylaxis of Plasmodium falciparum malaria in a human challenge model. Clin Infect Dis 54: 232-239.

53. Willerson D Jr., Rieckmann KH, Kass L, Carson PE, Frischer $\mathrm{H}$, Bowman JE, 1972. The chemoprophylactic use of diformyl diaminodiphenyl sulfone (DFD) in falciparum malaria. $\mathrm{Am} \mathrm{J}$ Trop Med Hyg 21: 138-143.

54. Willerson D Jr., Rieckmann KH, Carson PE, Frischer H, 1972. Effects of minocycline against chloroquine-resistant falciparum malaria. Am J Trop Med Hyg 21: 857-862.

55. Nyunt MM, Hendrix CW, Bakshi RP, Kumar N, Shapiro TA, 2009. Phase I/II evaluation of the prophylactic antimalarial activity of pafuramidine in healthy volunteers challenged with Plasmodium falciparum sporozoites. Am J Trop Med Hyg 80: 528-535.

56. Rieckmann KH, McNamara JV, Kass L, Powell RD, 1969. Gametocytocidal and sporontocidal effects of primaquine upon two strains of Plasmodium falciparum. Mil Med 134: 802-819.

57. Rieckmann KH, Trenholme GM, Williams RL, Carson PE, Frischer H, Desjardins RE, 1974. Prophylactic activity of mefloquine hydrochloride (WR 142490) in drug-resistant malaria. Bull World Health Organ 51: 375-377.

58. Murphy SC et al., 2017. A randomized trial of the prophylactic activity of DSM265 against pre-erythrocytic Plasmodium falciparum controlled human malaria infection by mosquito bites and direct venous inoculation. $J$ Infect Dis 217: 693-702.

59. Reuling IJ et al., 2018. A randomized feasibility trial comparing four antimalarial drug regimens to induce Plasmodium falciparum gametocytemia in the controlled human malaria infection model. Elife 7: e31549.

60. Talley AK et al., 2014. Safety and comparability of controlled human Plasmodium falciparum infection by mosquito bite in malaria-naive subjects at a new facility for sporozoite challenge. PLoS One 9: e109654.

61. Laurens MB et al., 2013. Successful human infection with $P$. falciparum using three aseptic Anopheles stephensi mosquitoes: a new model for controlled human malaria infection. PLoS One 8: e68969.

62. Lyke KE et al., 2010. Plasmodium falciparum malaria challenge by the bite of aseptic Anopheles stephensi mosquitoes: results of a randomized infectivity trial. PLoS One 5: e13490.

63. Roestenberg M, O'Hara GA, Duncan CJ, Epstein JE, Edwards NJ, Scholzen A, van der Ven AJ, Hermsen CC, Hill AV, Sauerwein $\mathrm{RW}, 2012$. Comparison of clinical and parasitological data from controlled human malaria infection trials. PLoS One 7: e38434.

64. Hermsen CC, de Vlas SJ, van Gemert GJ, Telgt DS, Verhage DF, Sauerwein RW, 2004. Testing vaccines in human experimental 
malaria: statistical analysis of parasitemia measured by a quantitative real-time polymerase chain reaction. Am J Trop Med Hyg 71: 196-201.

65. Langenberg MCC et al., 2018. Controlled Human malaria infection with graded numbers of Plasmodium falciparum NF135.C10- or NF166.C8-infected mosquitoes. Am J Trop Med Hyg 99: 709-712.

66. Gomez-Perez GP et al., 2015. Controlled human malaria infection by intramuscular and direct venous inoculation of cryopreserved Plasmodium falciparum sporozoites in malaria-naive volunteers: effect of injection volume and dose on infectivity rates. Malar $J$ 14: 306 .

67. Roestenberg M et al., 2013. Controlled human malaria infections by intradermal injection of cryopreserved Plasmodium falciparum sporozoites. Am J Trop Med Hyg 88: 5-13.

68. Sheehy SH et al., 2013. Optimising controlled human malaria infection studies using cryopreserved $P$. falciparum parasites administered by needle and syringe. PLoS One 8: e65960.

69. Shekalaghe $S$ et al., 2014. Controlled human malaria infection of Tanzanians by intradermal injection of aseptic, purified, cryopreserved Plasmodium falciparum sporozoites. Am J Trop Med Hyg 91: 471-480.

70. Bastiaens GJ et al., 2016. Safety, immunogenicity, and protective efficacy of intradermal immunization with aseptic, purified, cryopreserved Plasmodium falciparum sporozoites in volunteers under chloroquine prophylaxis: a randomized controlled trial. Am J Trop Med Hyg 94: 663-673.

71. Sulyok $\mathrm{M}$ et al., 2017. DSM265 for Plasmodium falciparum chemoprophylaxis: a randomised, double blinded, phase 1 trial with controlled human malaria infection. Lancet Infect Dis 17: 636-644.

72. Hodgson SH et al., 2014. Evaluating controlled human malaria infection in Kenyan adults with varying degrees of prior exposure to Plasmodium falciparum using sporozoites administered by intramuscular injection. Front Microbiol 5: 686.

73. Mordmuller B et al., 2015. Direct venous inoculation of Plasmodium falciparum sporozoites for controlled human malaria infection: a dose-finding trial in two centres. Malar J 14: 117.

74. Lyke KE et al., 2015. Optimizing intradermal administration of cryopreserved Plasmodium falciparum sporozoites in controlled human malaria infection. Am J Trop Med Hyg 93: 1274-1284.
75. Obiero JM et al., 2015. Impact of malaria preexposure on antiparasite cellular and humoral immune responses after controlled human malaria infection. Infect Immun 83: 2185-2196.

76. Hall CE et al., 2018. Mosquito-bite induced controlled human malaria infection with $P$. vivax or $P$. falciparum generates immune responses to homologous and heterologous preerythrocytic and erythrocytic antigens. Infect Immun 87: e00541-18.

77. Lell B et al., 2018. Impact of sickle cell trait and naturally acquired immunity on uncomplicated malaria after controlled human malaria infection in adults in Gabon. Am J Trop Med Hyg 98: 508-515.

78. Low LM et al., 2019. Controlled infection immunization using delayed death drug treatment elicits protective immune responses to blood-stage malaria parasites. Infect Immun 87: e00587-18.

79. Oh RC, Hustead TR, Ali SM, Pantsari MW, 2017. Mildly elevated liver transaminase levels: causes and evaluation. Am Fam Physician 96: 709-715.

80. Krishna S, Pukrittayakamee S, Supanaranond W, ter Kuile F, Ruprah M, Sura T, White NJ, 1995. Fever in uncomplicated Plasmodium falciparum malaria: randomized double-'blind' comparison of ibuprofen and paracetamol treatment. Trans $R$ Soc Trop Med Hyg 89: 507-509.

81. Rainsford KD, Bjarnason I, 2012. NSAIDs: take with food or after fasting? J Pharm Pharmacol 64: 465-469.

82. United States National Library of Medicine, Drug record: Ibuprofen. Available at: https://livertox.nlm.nih.gov/lbuprofen.htm. Accessed September 24, 2018.

83. Douros A, Bronder E, Klimpel A, Erley C, Garbe E, Kreutz R, 2018. Drug-induced kidney injury: a large case series from the Berlin case-control surveillance study. Clin Nephrol 89: 18-26.

84. Church RJ et al., 2019. Candidate biomarkers for the diagnosis and prognosis of drug-induced liver injury: an international collaborative effort. Hepatology 69: 760-773.

85. European Medicines Agency, 2016. Letter of Support for DrugInduced Liver Injury (DILI) Biomarker. Available at: https:// www.ema.europa.eu/en/documents/other/letter-support-druginduced-liver-injury-dili-biomarker_en.pdf. Accessed July 11, 2019.

86. Food and Drug Administration, 2016. Letter of Support for Drug-Induced Liver Injury (DJU) Biomarker(s) Available at: https:// www.fda.gov/media/99856/download. Accessed July 11, 2019.

87. Merz M, Lee KR, Kullak-Ublick GA, Brueckner A, Watkins PB, 2014. Methodology to assess clinical liver safety data. Drug Saf 37 (Suppl 1): S33-S45. 